\title{
Correlation energy for the homogeneous electron gas: Exact Bethe-Salpeter solution and an approximate evaluation
}

\author{
Emanuele Maggio and Georg Kresse* \\ University of Vienna, Faculty of Physics and Center for Computational Materials Science, Sensengasse 8/12, A-1090 Vienna, Austria
}

(Received 11 December 2015; revised manuscript received 7 April 2016; published 7 June 2016)

\begin{abstract}
The correlation energy of the homogeneous electron gas is evaluated by solving the Bethe-Salpeter equation (BSE) beyond the Tamm-Dancoff approximation for the electronic polarization propagator. The BSE is expected to improve on the random-phase approximation, owing to the inclusion of exchange diagrams. For instance, since the BSE reduces in second order to Møller-Plesset perturbation theory, it is self-interaction free in second order. Results for the correlation energy are compared with quantum Monte Carlo benchmarks and excellent agreement is observed. For low densities, however, we find imaginary eigenmodes in the polarization propagator. To avoid the occurrence of imaginary eigenmodes, an approximation to the BSE kernel is proposed that allows us to completely remove this issue in the low-electron-density region. We refer to this approximation as the random-phase approximation with screened exchange (RPAsX). We show that this approximation even slightly improves upon the standard BSE kernel.
\end{abstract}

DOI: 10.1103/PhysRevB.93.235113

\section{INTRODUCTION}

The study of the homogeneous electron gas (HEG) as a model in condensed matter theory, has a long tradition. In fact, it allows one to focus on the properties of the manyelectron system without complications arising from discretized lattice symmetry. Instead, the neutralizing positive charges are uniformly spread out and nonresponsive. Even if interactions among electrons are not present in the model, its pair correlation function is not constant. This is a consequence of the Pauli principle affecting same-spin electrons. The exchange energy introduced this way promotes a spin polarization within the electron gas and, at the same time, increases the electrons' kinetic energy [1]. As the Coulomb repulsion among electrons is turned on, correlations between particles (prevalently of opposite spin) [2] set in. This mechanism produces even stronger deviations from the free-electron case. One of the reasons for a continued interest in this model is that it provides density functional theory (DFT) with a natural starting point for the unknown exchange and correlation potential.

An attempt to supersede the mean-field description of the HEG dates back to the seminal work by Hubbard [3]. There, a local field factor was introduced to compute the system's response functions. Albeit progress has been made to evaluate this quantity, whose asymptotic behavior is known exactly [4], its complete characterization is still lacking [5]. On the other hand, the field factor can be related to the irreducible electronhole scattering amplitude. This appears in the integral (and recursive) equation for the polarization propagator, known as the Bethe-Salpeter equation (BSE). Therefore, by solving the BSE one can gain access to exchange and correlation properties. The BSE kernel $I$ itself is related to the irreducible

\footnotetext{
*georg.kresse@univie.ac.at
}

Published by the American Physical Society under the terms of the Creative Commons Attribution 3.0 License. Further distribution of this work must maintain attribution to the author(s) and the published article's title, journal citation, and DOI. self-energy through the relation $I=-\frac{1}{i} \frac{\delta \Sigma}{\delta G}$. Hence, the degree of sophistication in the BSE approach can be systematically improved by including more self-energy diagrams at the many-body perturbation theory (MBPT) level. In this study, we implement a computational scheme to solve the BSE when its kernel is derived from the $G W_{0}$ approximation for the self-energy. We then evaluate the correlation energy of the HEG and compare it against quantum Monte Carlo benchmarks.

The $G W_{0}$ and the random-phase approximation (RPA) include topologically equivalent energy diagrams [6]. In both cases, a subclass of self-energy diagrams, the so-called "bubble" diagrams, are summed up to infinite order [7]. While the RPA approach has witnessed alternate fortune over the years, there has been a strong resurgence of interest in the field recently [8-18]. Application of the RPA method to molecules [8,19-23] and solid-state systems [9,15,24-27] has highlighted its capabilities but also its limitations. The main advantage of RPA-related schemes stems from the seamless inclusion of long-range dispersion (typically not included in standard DFT potentials) [14]. On the other hand, systematic underestimation of binding energies [8,28,29] and nonphysical features for dissociation curves in diatomic systems [30,31] are some shortcomings of the RPA. To improve on this, the BSE has been exploited to describe hydrogen dissociation [32], to gain access to optical properties of semiconductors and insulators [33-35], and to study excitonic effects in extended [36] and molecular systems $[37,38]$. To the best of our knowledge, the vast majority of previous studies not concerned with exciton characterization has been carried out on DFT reference states; this is rectified herein.

To assess the correlation energy of the HEG, the adiabatic connection (AC) formalism will be employed in this study. From its inception, the AC formalism has been applied to obtain the correlation energy starting from reference states evaluated either by using Hartree-Fock [39] or Kohn-Sham [40-42] frameworks. In either case, the fluctuation-dissipation theorem is exploited to relate the ground-state correlation energy to the system's linear response functions integrated over the AC path. 
The study of the HEG is challenging for many reasons. Among others, correlations associated with large-momentumtransfer interactions [43] and between spin-parallel electrons [2] are overestimated by RPA. The computational scheme employed here addresses these issues by including screening effects and exchange contributions to infinite order in perturbation theory.

The paper is structured as follows: we first derive an expression for the correlation energy in terms of the polarization propagator. Then in Sec. II B the implementation of the BSE as a non-Hermitian generalized eigenvalue problem is described. Properties peculiar to the Bloch representation are discussed at the end of the theory section. Computational details are reported in Sec. III; finally, in Sec. IV, the computational scheme proposed and related approximations are put to fruition to assess the correlation energy of the HEG.

\section{THEORY}

\section{A. Exchange and correlation energy evaluated along the adiabatic-connection path}

The AC formalism was originally introduced in the DFT framework [40-42] to provide a compact expression for the exchange and correlation energy. In DFT, this quantity is given by the sum of two contributions: the electron-electron interaction and the difference in the kinetic energy between the "physical" system and the Kohn-Sham system. These terms are related to the two-body density matrix and to the one-body density matrix, respectively. Similarly, we can also decompose the exchange-correlation energy into one- and two-body contributions. The two-body part of the correlation energy can be estimated by solving the BSE, as described in Sec. II B. The impact of one-body contributions has been the subject of recent studies $[29,44]$. In this section we compactly derive an expression for the correlation energy and discuss the main assumptions required; the details are reported in Appendix A.

The system's Hamiltonian in second-quantized form along the AC path reads

$$
\begin{aligned}
\hat{\mathcal{H}}_{\lambda}= & \int d \mathbf{x} d \mathbf{x}^{\prime} \hat{\psi}^{\dagger}(\mathbf{x})\left\langle\mathbf{x}|\hat{h}| \mathbf{x}^{\prime}\right\rangle \hat{\psi}\left(\mathbf{x}^{\prime}\right) \\
& +\frac{\lambda}{2} \int d \mathbf{x} d \mathbf{x}^{\prime} v\left(\mathbf{x}, \mathbf{x}^{\prime}\right) \hat{\psi}^{\dagger}(\mathbf{x}) \hat{\psi}^{\dagger}\left(\mathbf{x}^{\prime}\right) \hat{\psi}\left(\mathbf{x}^{\prime}\right) \hat{\psi}(\mathbf{x}) \\
& +\int d \mathbf{x} d \mathbf{x}^{\prime} \hat{\psi}^{\dagger}(\mathbf{x})\left\langle\mathbf{x}\left|\hat{V}_{\lambda}^{\mathrm{xc}}\right| \mathbf{x}^{\prime}\right\rangle \hat{\psi}\left(\mathbf{x}^{\prime}\right) .
\end{aligned}
$$

The field operators $\hat{\psi}(\mathbf{x})$ and their adjoint are given in the ordinary Schrödinger picture; the Coulomb potential is defined in the spin-space basis as $v\left(\mathbf{x}, \mathbf{x}^{\prime}\right)=\delta_{\sigma, \sigma^{\prime}} /\left|\mathbf{r}-\mathbf{r}^{\prime}\right|$ with the usual spacetime notation $(\mathbf{x}, t)=(\mathbf{r}, \sigma, t)$ for the space $\mathbf{r}$, spin $\sigma$, and time $t$ variables. The one-particle operator $\hat{V}_{\lambda}^{\mathrm{xc}}$ can be any approximate exchange correlation potential, even one that is not diagonal in real space; for instance, a Hermitian energy-independent approximation for the self-energy $\Sigma_{\mathrm{xc}}(\omega)$.

Along the AC path the many-body interactions are progressively switched on, inducing changes in the correlations between electrons previously captured only by the exchangecorrelation potential $\hat{V}_{0}^{\mathrm{xc}}$. The latter also varies with varying coupling constant and different switching can be realized for
$\hat{V}_{\lambda}^{\mathrm{xc}}$. These are either designed to keep the electron density fixed along the AC path [41] or assume a linear dependence on $\lambda[45,46]$. Here we use the linear switching $\hat{V}_{\lambda}^{\mathrm{xc}}=(1-\lambda) \hat{V}_{0}^{\mathrm{xc}}$.

The correlation energy can be defined in full generality as the difference between the expectation values of the interacting Hamiltonian acting on its ground state and on the ground state of the noninteracting system:

$$
E_{c}=\left\langle\Psi_{1}\left|\hat{\mathcal{H}}_{1}\right| \Psi_{1}\right\rangle-\left\langle\Psi_{0}\left|\hat{\mathcal{H}}_{1}\right| \Psi_{0}\right\rangle
$$

If the symmetry of the ground state does not change along the AC path, then $\left|\Psi_{0}\right\rangle$ evolves into $\left|\Psi_{1}\right\rangle$ for nondegenerate ground states thanks to the Gell-Mann and Low theorem. It is easy to see that, with $E_{\lambda} \equiv\left\langle\Psi_{\lambda}\left|\mathcal{H}_{\lambda}\right| \Psi_{\lambda}\right\rangle,\left\langle\Psi_{0}\left|\mathcal{H}_{1}\right| \Psi_{0}\right\rangle=$ $E_{0}+\left\langle\Psi_{0}\left|\frac{1}{2} v-\hat{V}_{0}^{\mathrm{xc}}\right| \Psi_{0}\right\rangle$. Then the correlation energy can be expressed as (see also Refs. $[17,44]$ )

$$
E_{c}=E_{1}-E_{0}-\left\langle\Psi_{0}\left|\frac{\partial \mathcal{H}_{\lambda}}{\partial \lambda}\right| \Psi_{0}\right\rangle=\int_{0}^{1} d \lambda \frac{d E_{\lambda}}{d \lambda}-\left.\frac{d E_{\lambda}}{d \lambda}\right|_{\lambda=0}
$$

where we have assumed that the Hellmann-Feynman theorem holds in order to set $\frac{\partial}{\partial \lambda}\left\langle\Psi_{\lambda}\left|\mathcal{H}_{\lambda}\right| \Psi_{\lambda}\right\rangle=\left\langle\Psi_{\lambda}\left|\frac{\partial \mathcal{H}_{\lambda}}{\partial \lambda}\right| \Psi_{\lambda}\right\rangle$. The previous equation reproduces the standard expression found in the literature [17].

The calculation of the correlation energy thus requires evaluating the term $\frac{d E_{\lambda}}{d \lambda} \equiv \dot{E}_{\lambda}$ along the AC path, where the dependence on the coupling constant is retained by the ground-state wave function. The derivative of the ground-state energy can be written as

$$
\begin{aligned}
\frac{d E_{\lambda}}{d \lambda}= & \frac{1}{2} \iint d \mathbf{x} d \mathbf{x}^{\prime} v\left(\mathbf{x}, \mathbf{x}^{\prime}\right)\left\langle\psi^{\dagger}(\mathbf{x}) \psi^{\dagger}\left(\mathbf{x}^{\prime}\right) \psi\left(\mathbf{x}^{\prime}\right) \psi(\mathbf{x})\right\rangle_{\lambda} \\
& -\iint d \mathbf{x} d \mathbf{x}^{\prime}\left\langle\mathbf{x}^{\prime}\left|\hat{V}_{0}^{\mathrm{xc}}\right| \mathbf{x}\right\rangle\left\langle\psi^{\dagger}\left(\mathbf{x}^{\prime}\right) \psi(\mathbf{x})\right\rangle_{\lambda} .
\end{aligned}
$$

The last term in the previous equation represents contributions related to the change of the one-particle Green's function to the correlation energy $[29,44]$. In the present study, we disregard this term and concentrate on the first term on the right-hand side of Eq. (4). In fact, in second order, the last term is exactly zero for the HEG (see, e.g., chapter 22 in Ref. [47]), suggesting that its contribution should be small.

It is shown in Appendix A that the expression above can be recast in terms of the four-point polarization propagator $P_{\lambda}$ [48], fulfilling the BSE as detailed in the next section. The correlation energy then reads

$$
\begin{aligned}
E_{c}= & -\frac{1}{2} \int_{0}^{1} d \lambda \int \frac{d \omega}{2 \pi} \int \prod_{i=1}^{4} d \mathbf{x}_{i} v\left(\mathbf{x}_{1}, \mathbf{x}_{4}\right) \\
& \times \delta\left(\mathbf{x}_{4}-\mathbf{x}_{2}\right) \delta\left(\mathbf{x}_{3}-\mathbf{x}_{1}\right) \\
& \times\left[P_{\lambda}\left(\mathbf{x}_{1}, \mathbf{x}_{2}, \mathbf{x}_{3}, \mathbf{x}_{4} ; \omega\right)-P_{0}\left(\mathbf{x}_{1}, \mathbf{x}_{2}, \mathbf{x}_{3}, \mathbf{x}_{4} ; \omega\right)\right] .
\end{aligned}
$$

With the aid of the polarization propagator's spectral representation obtained in Appendix B, it is possible to carry out the frequency integration analytically, if the interparticle interaction is not frequency dependent [49]. 


\section{B. Bethe-Salpeter equation along the adiabatic-connection path}

The Bethe-Salpeter equation $P=P_{0}+P_{0} I P$ relates the fully interacting polarization propagator $P$ to the independent particle-hole propagator $P_{0}$, invoking an interaction kernel $I$. The kernel describes all possible interactions between particles and holes [50]. Solving this equation is generally a formidable task, which warrants the use of approximations. A commonly used one is to make the interaction kernel instantaneous and to include only Hartree and screened exchange interactions in the kernel $I(1,2,3,4)=V(1,2,3,4)-W_{0}(2,1,3,4)[33,34,51,52]$. By using the conventional spacetime notation (where the superscript "+" denotes positive infinitesimal time shifts), one can write

$$
\begin{aligned}
V(1,2,3,4) & =v\left(1,4^{+}\right) \delta\left(4,2^{+}\right) \delta\left(3,1^{+}\right), \\
W_{0}(1,2,3,4) & =w\left(1,4^{+}\right) \delta\left(4,2^{+}\right) \delta\left(3,1^{+}\right) .
\end{aligned}
$$

Here, $V$ and $W_{0}$ are the four-point generalizations of the bare Coulomb $v$ and screened $w$ interactions, and the shorthand $(i)=\left(\mathbf{r}_{i}, \sigma_{i}, t_{i}\right)$ refers to space, spin, and time degrees of freedom. The adiabatic switching of many-body interactions (presented in the previous section) requires the irreducible kernel to be linearly scaled by the coupling constant $\lambda$ and, correspondingly, the polarization propagator $P_{\lambda}$ to be evaluated for each point along the $\mathrm{AC}$ path. The resulting BSE will then be parametrically dependent on two quantities: the coupling constant $\lambda$ and the frequency variable $\omega$, provided that the kernel is static. This approximation for the kernel implies that $w\left(\mathbf{x}_{1}, \mathbf{x}_{2}\right)$ in Eq. (7) is evaluated as $w\left(\mathbf{x}_{1}, \mathbf{x}_{2}\right)=$ $\left\{v^{\frac{1}{2}} \epsilon^{-1}(\omega=0) v^{\frac{1}{2}}\right\}\left(\mathbf{x}_{1}, \mathbf{x}_{2}\right)$, where internal space variables are integrated over and the dielectric function in this study is computed at the RPA level.

It is convenient to introduce the "particle-hole" basis: $\Phi_{M}^{R}\left(\mathbf{x}, \mathbf{x}^{\prime}\right)=\psi_{a}(\mathbf{x}) \psi_{i}^{*}\left(\mathbf{x}^{\prime}\right)$ and $\Phi_{M}^{A}\left(\mathbf{x}, \mathbf{x}^{\prime}\right)=\psi_{i}(\mathbf{x}) \psi_{a}^{*}\left(\mathbf{x}^{\prime}\right)$, for the resonant $R$ and antiresonant $A$ component labeled by the "superindex" $M \equiv(i, a)$. The BSE can then be recast in this basis to yield

$$
\mathbf{P}_{\lambda}(\omega)=\mathbf{P}_{0}(\omega)+\mathbf{P}_{0}(\omega) * \mathbf{I}_{\lambda}(\omega=0) * \mathbf{P}_{\lambda}(\omega) .
$$

The symbol " $*$ " indicates the usual matrix product in the chosen orbital basis. The independent particle-hole propagator is diagonal in this basis and can be expressed as

$$
P_{0}\left(\mathbf{x}_{1}, \mathbf{x}_{2}, \mathbf{x}_{1}^{\prime}, \mathbf{x}_{2}^{\prime} ; \omega\right)=\operatorname{tr}\left\{\mathbf{P}_{0}(\omega)\right\}=\sum_{i, a} f_{i} \bar{f}_{a}\left(\frac{\psi_{a}\left(\mathbf{x}_{1}\right) \psi_{i}^{*}\left(\mathbf{x}_{1}^{\prime}\right) \psi_{i}\left(\mathbf{x}_{2}\right) \psi_{a}^{*}\left(\mathbf{x}_{2}^{\prime}\right)}{\omega-\Delta E_{i a}}+\frac{\psi_{i}\left(\mathbf{x}_{1}\right) \psi_{a}^{*}\left(\mathbf{x}_{1}^{\prime}\right) \psi_{a}\left(\mathbf{x}_{2}\right) \psi_{i}^{*}\left(\mathbf{x}_{2}^{\prime}\right)}{-\omega-\Delta E_{i a}}\right)
$$

where $f_{n}$ is the (fractional) occupancy for the orbital $n$ and $\bar{f}_{n}=1-f_{n}$; the indices $i$ and $a$ in the summations above go over the occupied and unoccupied single-particle states, respectively. $\Delta E_{i a}=\left(\epsilon_{a}-\epsilon_{i}\right)$ is the independent-particle energy difference.

Since the BSE kernel is static, it is possible to invert the matrix equation (8) for each frequency point. In Appendix B it is shown that this is, however, not necessary since the spectral decomposition for the polarization propagator $\mathbf{P}_{\lambda}(\omega)$ can be constructed by solving the non-Hermitian generalized eigenvalue problem (EVP)

$$
\left(\begin{array}{cc}
\mathbf{A} & \mathbf{B} \\
\mathbf{B}^{*} & \mathbf{A}^{*}
\end{array}\right)\left(\begin{array}{l}
\mathbf{X} \\
\mathbf{Y}
\end{array}\right)=\left(\begin{array}{cc}
\Omega & \mathbf{0} \\
\mathbf{0} & -\Omega
\end{array}\right)\left(\begin{array}{l}
\mathbf{X} \\
\mathbf{Y}
\end{array}\right)
$$

which does not depend on $\omega$. The matrix elements for $\mathbf{A}$ and $\mathbf{B}$ are given by [53]

$$
\begin{gathered}
A_{i a j b}=\left(\Delta E_{i a}\right)+\lambda\langle a j|V| i b\rangle-\lambda\langle a j|W| b i\rangle, \\
B_{i a j b}=\lambda\langle a b|V| i j\rangle-\lambda\langle a b|W| j i\rangle .
\end{gathered}
$$

Their time-ordered diagrammatic representation is shown in Fig. 1.

The dimension of this non-Hermitian eigenvalue problem is given by the sum of particle-hole and hole-particle transitions. It is possible, however, to reduce the matrix size to a single particle-hole subspace by exploiting the time-reversal symmetry of the system. This statement will be proved in the next section where we introduce a suitable basis for the matrix representation as in Ref. [54].

\section{Bloch representation}

For extended systems it is convenient to switch to a reciprocal-space representation of the electronic problem to capture more readily its translational invariance. A fully homogeneous system has the highest translational symmetry, which translates into a spherical Fermi surface. In this study, we choose to discretize the infinite, translational invariant system into a unit cell of given symmetry and subject to periodic boundary conditions. This approach is akin to the QMC benchmarks against which we will compare the outcome of our calculations. As a consequence, this discretization changes the Fermi surface, which is now replaced by a convex polyhedron: this tends to the exact Fermi surface as the number of unit-cell replicas (i.e., k-point sampling the reciprocal space) in our calculations is increased. Each single-particle quantum number $a, b, i, j$ can be mapped into a set specifying the particles' band (valence band for the hole states and conduction band for particle states) as well as their Bloch wave vectors, $\mathbf{k}, \mathbf{k}^{\prime}$. We have

$$
\left\{\begin{array}{l}
a \rightarrow\left(a, \mathbf{k}^{\prime}\right), \quad i \rightarrow\left(i, \mathbf{k}^{\prime} \pm \mathbf{q}\right) \\
b \rightarrow(b, \mathbf{k}), \quad j \rightarrow(j, \mathbf{k} \pm \mathbf{q})
\end{array}\right.
$$

where $\mathbf{q}$ is the momentum of the photon impinging the system and the \pm sign applies to resonant and antiresonant electronhole pairs, respectively. We denote the Fourier components of the Coulomb potential with $v_{\mathbf{q}}\left(\mathbf{r}, \mathbf{r}^{\prime}\right)$, then the two electron integrals in $\mathbf{A}$ are given by integrating over spin $\sigma, \varsigma$ and space variables 


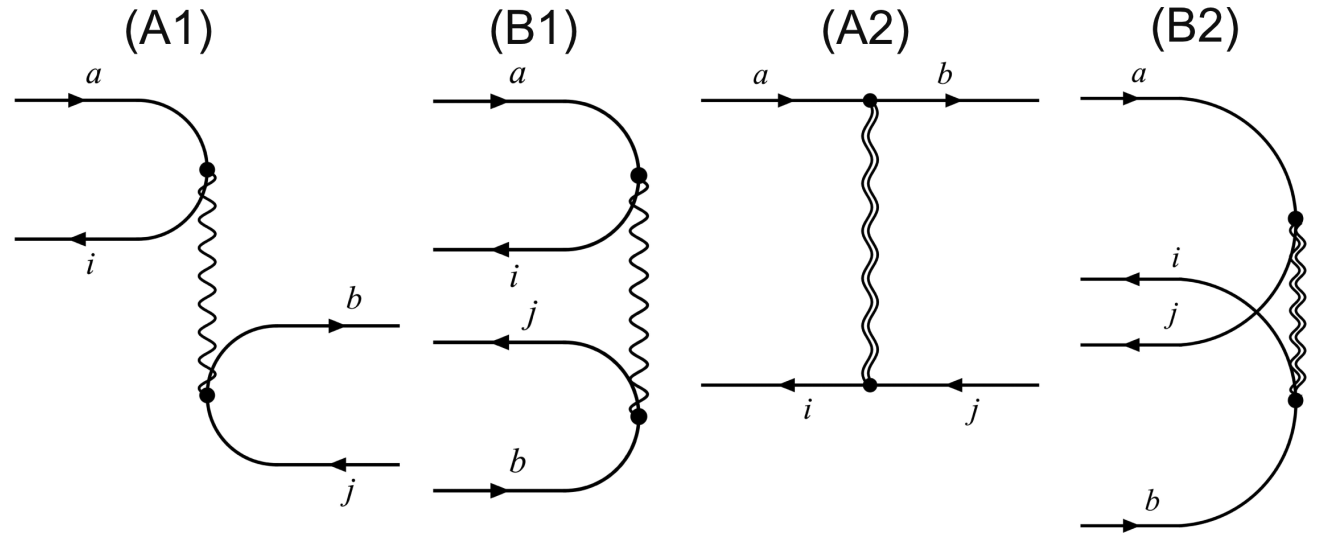

FIG. 1. Goldstone diagrams for the particle-hole interaction included in the BSE kernel: particle states are shown as right arrows $\rightarrow$ (labelled by unoccupied ground-state orbitals $a, b$ ) and holes are shown by left arrows $\leftarrow$ (labelled by occupied orbitals $i, j$ ). The bare and screened Coulomb interaction is represented by a simple wavy line and a double wavy line, respectively. Diagrams (A1) and (B1) represent the resonant and antiresonant particle-hole annihilation and simultaneous creation, whereas diagrams (A2) and (B2) represent the resonant and antiresonant particle-hole scattering process.

$$
\begin{aligned}
\langle a j|V| i b\rangle= & \sum_{\substack{\sigma, \sigma^{\prime} \\
\varsigma, \varsigma^{\prime}}} \int d \mathbf{r} d \mathbf{r}^{\prime} \psi_{a, \mathbf{k}^{\prime}}^{*}(\mathbf{r}, \sigma) \psi_{j, \mathbf{k}+\mathbf{q}}^{*}\left(\mathbf{r}^{\prime}, \varsigma^{\prime}\right) v_{\mathbf{q}}\left(\mathbf{r}, \mathbf{r}^{\prime}\right) \\
& \times \psi_{i, \mathbf{k}^{\prime}+\mathbf{q}}(\mathbf{r}, \varsigma) \psi_{b, \mathbf{k}}\left(\mathbf{r}^{\prime}, \sigma^{\prime}\right) \delta_{\sigma, \varsigma^{\prime}} \delta_{\sigma^{\prime}, \varsigma^{\prime}}, \\
\left\langle a j\left|W_{0}\right| b i\right\rangle= & \sum_{\substack{\sigma, \sigma^{\prime} \\
\varsigma, \varsigma^{\prime}}} \int d \mathbf{r} d \mathbf{r}^{\prime} \psi_{a, \mathbf{k}^{\prime}}^{*}(\mathbf{r}, \sigma) \psi_{j, \mathbf{k}+\mathbf{q}}^{*}\left(\mathbf{r}^{\prime}, \varsigma^{\prime}\right) w_{\mathbf{q}}\left(\mathbf{r}, \mathbf{r}^{\prime}\right) \\
& \times \psi_{b, \mathbf{k}}\left(\mathbf{r}, \sigma^{\prime}\right) \psi_{i, \mathbf{k}^{\prime}+\mathbf{q}}\left(\mathbf{r}^{\prime}, \varsigma\right) \delta_{\sigma, \sigma^{\prime}} \delta_{\zeta, \varsigma^{\prime}},
\end{aligned}
$$

and similarly for the $\mathbf{B}$ matrix elements:

$$
\begin{aligned}
\langle a b|V| i j\rangle= & \sum_{\substack{\sigma, \sigma^{\prime} \\
\varsigma, \varsigma^{\prime}}} \int d \mathbf{r} d \mathbf{r}^{\prime} \psi_{a, \mathbf{k}^{\prime}}^{*}(\mathbf{r}, \sigma) \psi_{b, \mathbf{k}}^{*}\left(\mathbf{r}^{\prime}, \sigma^{\prime}\right) v_{\mathbf{q}}\left(\mathbf{r}, \mathbf{r}^{\prime}\right) \\
& \times \psi_{i, \mathbf{k}^{\prime}+\mathbf{q}}(\mathbf{r}, \varsigma) \psi_{j, \mathbf{k}-\mathbf{q}}\left(\mathbf{r}^{\prime}, \varsigma^{\prime}\right) \delta_{\sigma, \varsigma^{\prime}} \delta_{\sigma^{\prime}, \varsigma^{\prime}}, \\
\left\langle a b\left|W_{0}\right| j i\right\rangle= & \sum_{\substack{\sigma, \sigma^{\prime} \\
\varsigma, \varsigma^{\prime}}} \int d \mathbf{r} d \mathbf{r}^{\prime} \psi_{a, \mathbf{k}^{\prime}}^{*}(\mathbf{r}, \sigma) \psi_{b, \mathbf{k}}^{*}\left(\mathbf{r}^{\prime}, \sigma^{\prime}\right) w_{\mathbf{q}}\left(\mathbf{r}, \mathbf{r}^{\prime}\right) \\
& \times \psi_{j, \mathbf{k}-\mathbf{q}}\left(\mathbf{r}, \varsigma^{\prime}\right) \psi_{i, \mathbf{k}^{\prime}+\mathbf{q}}\left(\mathbf{r}^{\prime}, \varsigma\right) \delta_{\sigma, \varsigma^{\prime}} \delta_{\sigma^{\prime}, \zeta}
\end{aligned}
$$

For the Hartree components in Eqs. (14) and (16), the interaction potential comprises of a single mode $\mathbf{q}$ equal to the difference $\mathbf{k}-\mathbf{k}^{\prime}$. Whereas the screened Coulomb interaction in Eqs. (15) and (17) can be decomposed in its Fourier modes,

$$
w_{\mathbf{q}}\left(\mathbf{r}, \mathbf{r}^{\prime}\right)=4 \pi e^{2} \sum_{\mathbf{G}, \mathbf{G}^{\prime}} \frac{e^{-\mathrm{i}(\mathbf{q}+\mathbf{G}) \cdot \mathbf{r}^{\prime}} \epsilon_{\mathbf{q}+\mathbf{G}, \mathbf{q}+\mathbf{G}^{\prime}} e^{\mathrm{i}\left(\mathbf{q}+\mathbf{G}^{\prime}\right) \cdot \mathbf{r}}}{\left|\mathbf{q}+\mathbf{G} \| \mathbf{q}+\mathbf{G}^{\prime}\right|} .
$$

The spin structure of the matrix elements in Eqs. (14), (16), and (17) spans the singlet subspace, where electron and hole states of each pair have the same spin. The exchange part in the A matrix [Eq. (15)] is defined in principle also on the triplet subspace where the incoming electron and hole have opposite spin orientations [34]. If the Hamiltonian is time-reversal invariant (i.e., spin-orbit coupling is not present) then the singlet and triplet solutions factorize [55] and only the former is required to evaluate optical properties and the correlation energy.

We can now move on to prove that, thanks to the timereversal symmetry of the wave function, the original EVP can be downfolded into a Hermitian EVP. The time-reversal property of the Schrödinger equation implies that, for a singleparticle state, the Bloch functions observe $\psi_{n,-\mathbf{k}}(\mathbf{r})=\psi_{n, \mathbf{k}}^{*}(\mathbf{r})$ and the one-electron eigenvalues are also the same. Since the basis functions spanning the space of resonant and antiresonant components are linearly independent, one can selectively invert the single particles' wave vectors in the antiresonant component as follows:

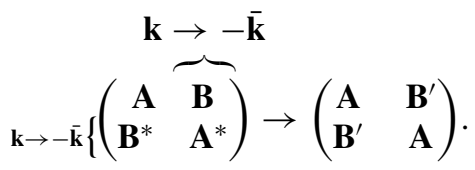

It is well known that the $\mathbf{A}$ matrix is Hermitian in this representation [56], hence the transformation above will simply map $\mathbf{A}^{*}$ into $\mathbf{A}$. For the exchange part in Eq. (17), the transformed matrix elements in $\mathbf{B}^{\prime}$ are given by (with the replacements $\left.\psi_{j,-\overline{\mathbf{k}}-\mathbf{q}} \rightarrow \psi_{j, \overline{\mathbf{k}}+\mathbf{q}}^{*}, \psi_{b,-\overline{\mathbf{k}}}^{*} \rightarrow \psi_{b, \overline{\mathbf{k}}}\right)$

$$
\int d \mathbf{r} d \mathbf{r}^{\prime} \psi_{a, \mathbf{k}^{\prime}}^{*}(\mathbf{r}) \psi_{j, \mathbf{k}+\mathbf{q}}^{*}(\mathbf{r}) w_{\mathbf{q}}\left(\mathbf{r}, \mathbf{r}^{\prime}\right) \psi_{b, \mathbf{k}}\left(\mathbf{r}^{\prime}\right) \psi_{i, \mathbf{k}^{\prime}+\mathbf{q}}\left(\mathbf{r}^{\prime}\right) .
$$

Here, we have dropped the bar over the $\mathbf{k}$ index and suppressed the spin indices for simplicity. The Hartree component turns out to be identical to the expression in Eq. (14), hence requiring no extra computational step.

Furthermore, the transformation applied to $\mathbf{B}^{*}$ gives exactly the same matrix elements in Eq. (20), as we now show. Starting with the matrix elements in Eq. (17) and applying the transformation $\mathbf{k}^{\prime} \rightarrow-\overline{\mathbf{k}}^{\prime}$ to the antiresonant electron-hole pair $(a, i)$, one obtains

$$
\begin{aligned}
\left\langle\bar{a} b\left|W_{0}\right| j \bar{i}\right\rangle^{*}= & \int d \mathbf{r} d \mathbf{r}^{\prime} \psi_{j, \mathbf{k}+\mathbf{q}}^{*}(\mathbf{r}) \psi_{i,-\overline{\mathbf{k}}^{\prime}-\mathbf{q}}^{*}\left(\mathbf{r}^{\prime}\right) \\
& \times w_{\mathbf{q}}^{*}\left(\mathbf{r}, \mathbf{r}^{\prime}\right) \psi_{a,-\overline{\mathbf{k}}^{\prime}}(\mathbf{r}) \psi_{b, \mathbf{k}}\left(\mathbf{r}^{\prime}\right) .
\end{aligned}
$$


In the static approximation to the screening potential $w_{\mathbf{q}}\left(\mathbf{r}, \mathbf{r}^{\prime}\right)$, the dielectric function at the RPA level is an even function of $|\mathbf{q}|$ and has zero imaginary part, hence it is possible to replace $w_{\mathbf{q}}^{*}\left(\mathbf{r}, \mathbf{r}^{\prime}\right)$ with $w_{-\mathbf{q}}\left(\mathbf{r}, \mathbf{r}^{\prime}\right)$ in the equation above. Finally, by swapping the integration variables, it is easy to see that the exchange component in $\mathbf{B}^{*^{\prime}}$ reduces to Eq. (20). The reasoning above can be straightforwardly repeated for the Hartree component in $\mathbf{B}^{*}$ to give $\langle a j|V| i b\rangle$. This result completes the proof of Eq. (19). In the following we will drop the prime when referring to the $\mathbf{B}^{\prime}$ matrix.

We can now proceed to reduce the dimensionality of the EVP (10). Adding and subtracting the individual equations contained in Eq. (10) and then solving one of the two equations, let us say with respect to $(\mathbf{X}-\mathbf{Y})$, one obtains the squared problem [57]:

$$
(\mathbf{A}+\mathbf{B})(\mathbf{A}-\mathbf{B})(\mathbf{X}+\mathbf{Y})=\Omega^{2}(\mathbf{X}+\mathbf{Y}) .
$$

The expression above can be converted into a conventional EVP if the matrices are positive definite. Then the matrix $(\mathbf{A}-\mathbf{B})^{\frac{1}{2}}$ is unique and the eigenvalue problem finally reads

$$
\begin{aligned}
& (\mathbf{A}-\mathbf{B})^{\frac{1}{2}}(\mathbf{A}+\mathbf{B})(\mathbf{A}-\mathbf{B})^{\frac{1}{2}}(\mathbf{A}-\mathbf{B})^{\frac{1}{2}}(\mathbf{X}+\mathbf{Y}) \\
& =\Omega^{2}(\mathbf{A}-\mathbf{B})^{\frac{1}{2}}(\mathbf{X}+\mathbf{Y}) .
\end{aligned}
$$

The difference $\mathbf{P}_{\lambda}(\omega)-\mathbf{P}_{0}(\omega)$ in Eq. (5) can be integrated over $\omega$ thanks to the spectral decomposition in Eq. (9) and in Eq. (B8). The correlation energy can then be expressed by using the correlation part of the two-particle density matrix $\mathcal{P}_{\lambda}$ :

$$
E_{c}=\frac{1}{2} \int_{0}^{1} d \lambda \operatorname{tr}\left\{\mathbf{V} \mathcal{P}_{\lambda}\right\},
$$

with $\mathcal{P}_{\lambda}=(\mathbf{X}+\mathbf{Y})_{\lambda}(\mathbf{X}+\mathbf{Y})_{\lambda}^{*}-\mathbf{1}$ and $\mathbf{V}$ being the Coulomb matrix in Eq. (14) [8,58].

\section{COMPUTATIONAL DETAILS}

The HEG is in principle specified by the average electron density $n=N_{e} / V$ (with $V$ being the unit-cell volume and $N_{e}$ the number of electrons in it) and the number of electrons in a given spin configuration (either $N_{e, \uparrow}$ or $N_{e, \downarrow}$ ). These quantities are easily translated into the Wigner-Seitz radius $r_{s}$ given by $\frac{4}{3} \pi r_{s}^{3}=V / N_{e}$ and the spin polarization $\zeta=$ $\left|N_{e, \uparrow}-N_{e, \downarrow}\right| / N_{e}$. The discretization of the Bloch wave vectors introduced in Sec. II C, however, requires also a specific choice for the k-point sampling and for the symmetry of the simulation cell.

Calculations were performed on a $\Gamma$-point centered, uniform, k-point mesh of dimensions $N_{k} \times N_{k} \times N_{k}$. Furthermore, we applied simple cubic unit cells in all our simulations. As already emphasized before, results are independent of the choice of the unit cell once k-point convergence is reached. We confirmed, for instance, that a face-centered cubic cell and a simple cubic cell result in the same RPA energy. Simple cubic cells are, however, often used for quantum Monte Carlo simulations. For instance, a $3 \times 3 \times 3 \mathbf{k}$-point grid with two electrons in the unit cell corresponds exactly to a $3 \times 3 \times 3$ super cell with 54 electrons, which was often used in QMC simulations because the resultant electronic configuration has only fully occupied or entirely unoccupied one-electron orbitals [59].
The VASP code $[60,61]$ has been used for all calculations, which required a three-stage computational procedure. The one-body Green's function variation was disregarded along the coupling-constant integral, as discussed above. The solidity of this approximation will be checked by comparing the resulting correlation energy against the QMC benchmark. For a given $\left(r_{s}, \zeta\right)$, the procedure to evaluate the correlation energy starts with a self-consistent calculation at the Kohn-Sham level. The plane-wave basis set is specified by the energy cutoff $E_{\text {cut }}$ and includes plane waves with kinetic energy smaller than the given cutoff value. This step is followed by a self-consistent evaluation of the quasiparticle energies at the $Q P-G W_{0}$ level $[62,63]$. The screening potential $W_{0}$ is evaluated at the RPA level with orbitals and eigenvalues from the previous DFT calculation. To ensure consistency of the results with previous calculations [25] for the evaluation of the correlation energy we set the cutoff for the response function's basis set (ENCUTGW) and densities in the two-electron integrals to the same value $E_{\text {cut }}$. The frequency sampling has been carried out on a linear grid, specified by its maximum value, set equal to $1.5 E_{\text {cut }}$ for all $\left(r_{s}, \zeta\right)$. The grid-point density has been chosen as 0.1 for $r_{s}=0.5,0.8$ and 0.2 for $r_{s}=1.0$ (collectively referred as the high-density region), then progressively increased to 0.6 and 1.0 in the intermediate $\left(r_{s}=2.0,3.0\right)$ and low densities $\left(r_{s}=4.0,5.0\right)$, respectively. The evaluation of quasiparticle energies, necessary to specify the dressed propagator, has been performed iteratively. We set the number of iterations to three; the resulting quasiparticle energies have a mean accuracy of $\approx 1 \mathrm{mRy}$. These are the only quantities being updated (plane waves being exact eigenfunctions for the HEG). Thus, the one-body Green's function retains a simple one-electron form.

The BSE was recast as in Eq. (22) and has been implemented in VASP; all virtual unoccupied orbitals spanned by the plane-wave basis were included. The diagonalization of Eq. (22) is required for each value of the coupling constant, whereas the Coulomb kernel (constant along the AC path) is computed and stored once and for all at the beginning of the BSE step. The integral in Eq. (23) is evaluated numerically with a Gauss-Legendre quadrature scheme. As few as two points are sufficient to produce results converged within the order of meV.

\section{RESULTS AND DISCUSSION}

We start by considering the correlation energy's convergence properties for a given $\left(r_{s}, \zeta\right)$ against the total number of plane waves. The impact of the basis-set incompleteness is shown in Fig. 2 (left panel): for the set of densities considered, a linear convergence behavior with the inverse of the number of bands is observed [14]. The complete basis-set limit has been estimated for both the paramagnetic $(\zeta=0)$ and ferromagnetic $(\zeta=1)$ case with a linear regression. For the ferromagnetic system a particularly fast convergence is observed as a function of the number of plane waves. To the best of our knowledge, an analytical wave-vector analysis is not available for arbitrary spin polarizations. However, in Ref. [64], the pair-correlationfunction limit for large wave vectors is said to be determined by the kinks in the many-electron wave function. For fully spin-polarized systems these are completely absent; this then 

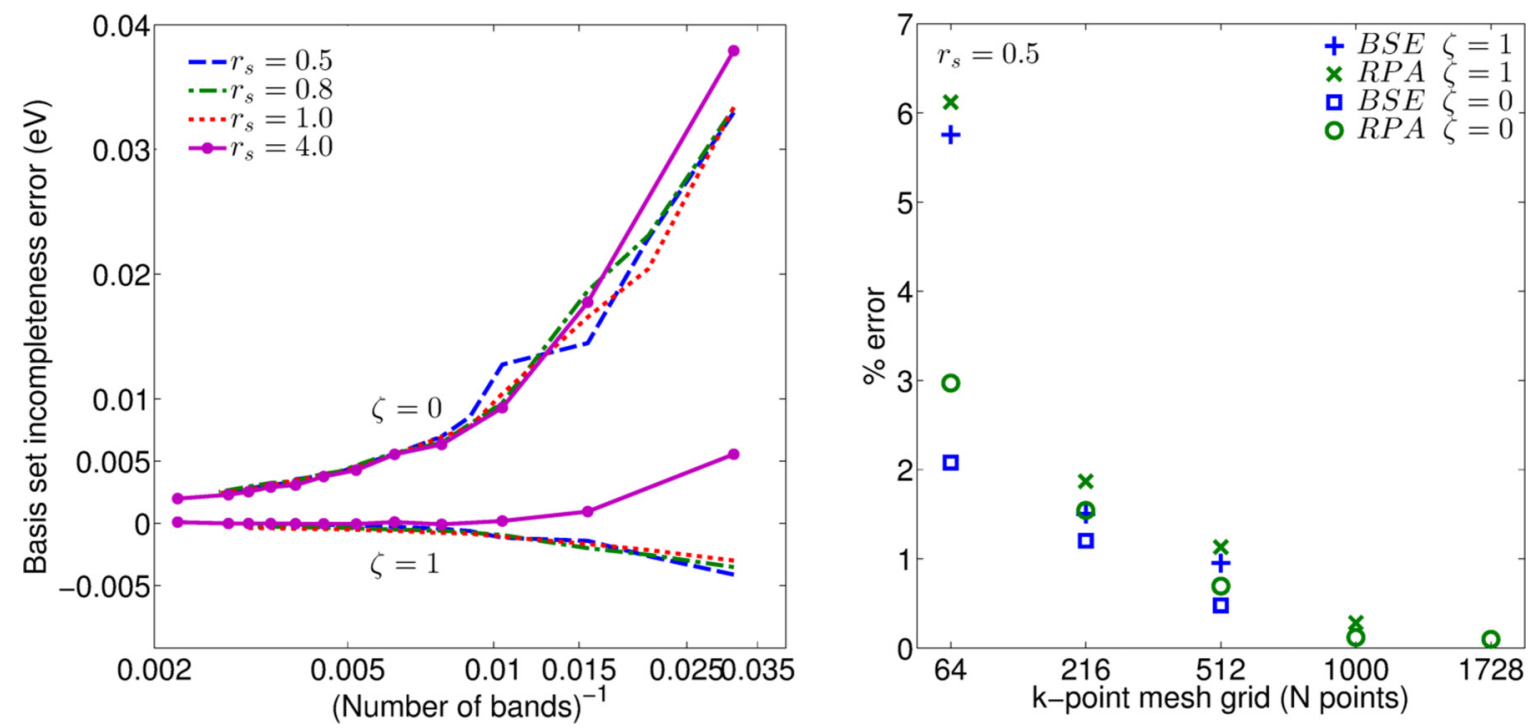

FIG. 2. (left panel) Convergence for the BSE correlation energy as a function of the total number of plane waves; the energy zero has been set equal to the linearly extrapolated complete-basis-energy limit for each value of $\left(r_{s}, \zeta\right)$. (right panel) Total energy convergence as a function of the number of points included in the reciprocal-space sampling. Here, the value shown is calculated by taking the difference between the total energy for any given $\mathbf{k}$ mesh and the value at the preceding sampling.

rationalizes the fast convergence observed. We will comment on this point in more detail below.

The assessment of the total energy convergence with respect to the k-point grid density is reported in the right panel of Fig. 2. Here, we report the total energy for any given $\mathbf{k}$-point mesh minus the value at the preceding k-point sampling divided by the energy obtained with the most accurate k-point grid for a given level of theory. For each k-point grid, the total energy has been computed on the $G W_{0}$ reference state and includes the one-electron kinetic energy, the exact exchange energy, a correction term related to the occurrence of fractional occupancies (Ref. [15]), as well as the correlation contribution. We include in the figure also the RPA energy convergence that provides an upper bound on the relative error. The corresponding error on the total energy is less than $1 \%$ for the ferromagnetic system and less than $0.5 \%$ for the paramagnetic ground state. On the other hand, convergence of the correlation energy alone proves more difficult. To estimate the correlation energy convergence with respect to both the basis set and the k-point sampling, we employ an extrapolation scheme adapted from Ref. [65]:

$$
E_{c}^{\infty}\left(N_{k} \rightarrow \infty\right) \approx E_{c}^{r}\left(N_{k} \rightarrow \infty\right)-E_{c}^{r}\left(N_{k}\right)+E_{c}^{\infty}\left(N_{k}\right),
$$

where the superscript of the correlation energy $E_{c}$ indicates the dependence on the basis set. The symbol $r$ indicates that the calculations were performed by using a small number of bands (32 bands here), whereas $\infty$ indicates that the results have been extrapolated to the infinite-basis-set limit. In practice, we found that it is sufficient to determine the basis set correction $\left[-E_{c}^{r}\left(N_{k}\right)+E_{c}^{\infty}\left(N_{k}\right)\right]$ by using $N_{k}=3$, i.e., $3 \times 3 \times 3 \mathbf{~ k}$ points.

To validate this somewhat involved approach, we first calculated the correlation energy starting from the Kohn-Sham ground state, i.e., RPA @ LDA. This should reproduce the previously published analytic results of Ref. [43] for the paramagnetic system and of Ref. [66] for the spin-polarized case. We tested the agreement for the case $\left(r_{s}=1, \zeta=0\right)$ where we found it necessary to include up to $N_{k}=18$ points to reproduce the analytical results within $4 \mathrm{meV}$. Fewer $\mathbf{k}$ points $\left(N_{k}=16\right)$ are necessary for the spin-polarized case to reproduce the data with a similar accuracy. We stress that the RPA results reported below have been calculated on the $G W_{0}$ reference and hence cannot match the historical RPA correlation energies, owing to the renormalization of the propagator lines in the RPA response function.

Given the current computational limitations, we adapt the extrapolation scheme above to the case of post-RPA calculations with the replacement

$$
E_{c}^{r}\left(N_{k} \rightarrow \infty\right) \approx E_{c}^{r}\left(N_{k}=8\right)+\Delta_{N_{k}},
$$

where the correlation energy is computed at the considered level of theory whereas the $\Delta_{N_{k}}$ correction corrects for the k-point incompleteness error. This value is difficult to calculate accurately for the BSE. In previous work [67], it was found that the second-order exchange reduces the direct correlation energies by about $1 / 3$. The situation is similar here, with exchange contributions reducing the correlation energy by roughly $30 \%$. In line with this, the k-point errors are generally $1 / 3$ smaller for BSE than for RPA (compare Fig. 2, but note that the left panel presents relative errors). Overall, it therefore seems sensible to determine the k-point error by using the computationally efficient RPA, but to reduce the RPA k-point correction by a factor $\frac{2}{3}$ if exchange is included.

The correlation energies computed for a set of $\left(r_{s}, \zeta\right)$ are shown in Fig. 3 by a solid line and compared against the quantum Monte Carlo estimates by Ceperly and Adler (CA in the following, indicated by circles in the figure) [68] and by Ortiz and Ballone (OB in the following, " $\times$ " symbols in the figure) [69]. Finite-size effects also impact these benchmarks [70] and different extrapolation schemes, which 

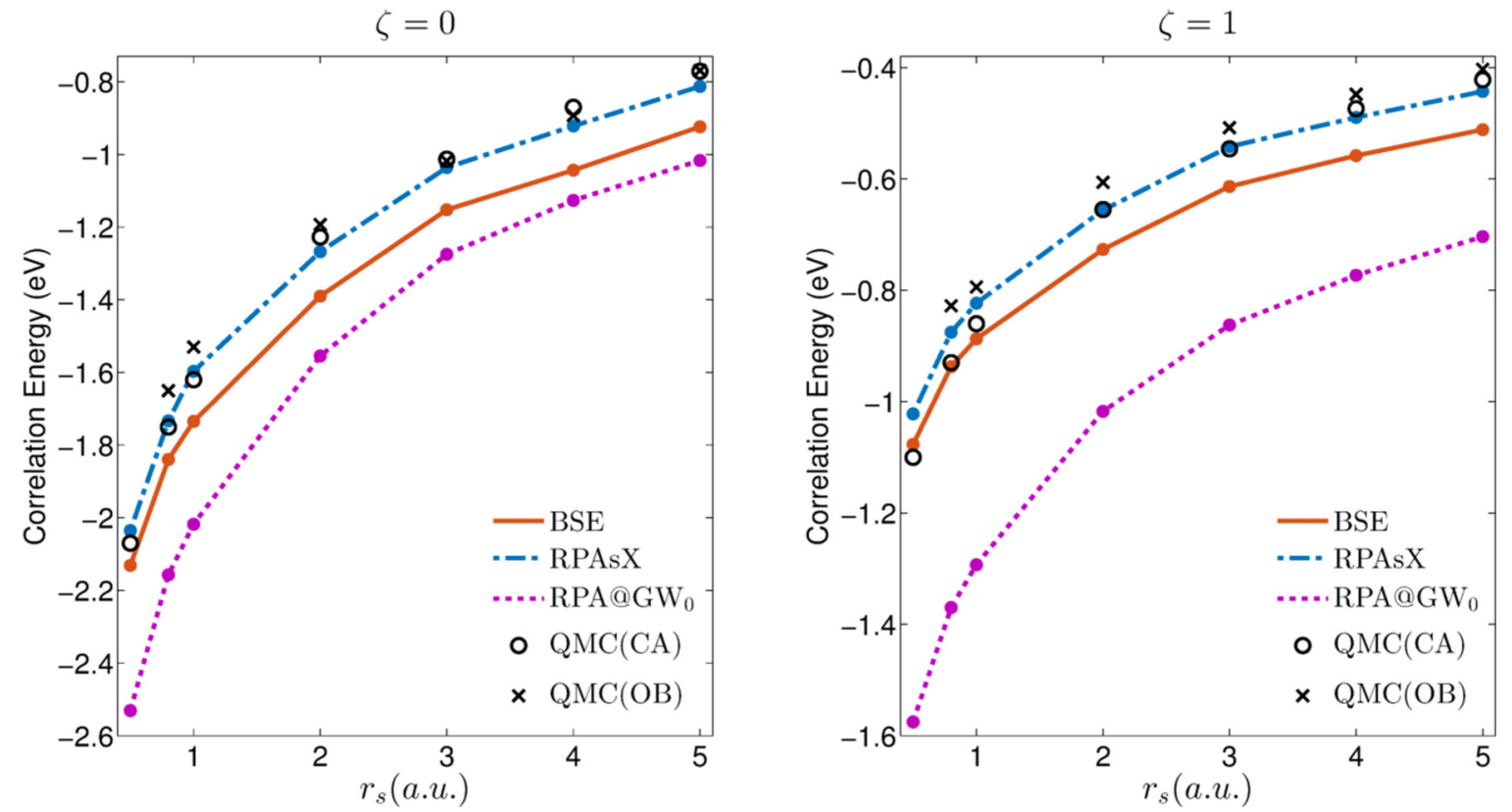

FIG. 3. Values of correlation energy for the full BSE (solid line) and for the RPAsX approximation (dash-dotted line) as a function of the Wigner-Seitz radius for the paramagnetic (left) and ferromagnetic (right) HEG. Results are compared against the QMC simulations in Ref. [68] (round symbols) and in Ref. [69] (crosses). The random-phase approximation on the same reference state (broken line) is also included.

do not necessarily fulfill the variational principle, have been employed. Since we did not want to judge which calculation is more accurate, we compare with both results. The RPA@ $G W_{0}$ estimate of the correlation energy (dotted line in Fig. 3), is obtained as usual by including the bubble diagrams only, i.e., excluding the ladder diagrams (A2) and (B2) in Fig. 1. The newly proposed RPA with screened exchange (dash-dotted line), where only bubbles and diagrams (B2) are included is also shown (see below). Starting our analysis from the full BSE calculations (solid line in Fig. 3), in the paramagnetic case the $\mathrm{CA}$ results are reproduced with a mismatch of $0.06 \mathrm{eV}$ for the highest density. The error progressively increases in the high-density region and becomes significant with a deviation of $\approx 20 \%$ for $r_{s} \geqslant 4.0$ a.u. This pattern is also observed for the ferromagnetic system, with the absolute deviation, however, decreasing to $\approx 10 \%$ at low densities.

The general trend of the present BSE results can be rationalized as follows: in the low-density region, the BSE makes a sizable error, which is of unknown origin but could be related to the lack of particle-particle (or hole-hole) ladder diagrams to describe short-range interactions [71]. Indeed, for the paramagnetic system (where short-range interactions are most important) we observe that the BSE and RPA results are close in energy and both deviate from the QMC estimate. In the high-density region the BSE reproduces well the QMC estimates for both values of spin polarization.

Short-range interactions are small in the ferromagnetic case owing to the Pauli exclusion principle and are incorrectly included by the RPA. This self-correlation error degrades the agreement between the QMC estimates and the RPA [72] (see left panel in Fig. 3). The self-correlation error is canceled in leading order by the BSE, as we discuss now. In the low-density region the large wave-vector contributions to the correlation energy become predominant [1]. At large wave vectors, the screened interaction $W_{0}$ equals the bare interaction $V$, since screening is weak. The leading contribution to the BSE then becomes equal to the second-order contribution in Møller-Plesset perturbation theory (MP2) given in Ref. [18]. This can be shown by determining the leading term in the correlation part of the two-body density matrix. For the Hartree term and the RPA, the equivalence has also been derived in Ref. [67]. In second order, the correlation energy is then simply given by the standard textbook equation:

$$
E_{c}^{(2)}=\frac{1}{2} \sum_{i j a b} \frac{\langle i j \mid a b\rangle}{\Delta E_{i a}+\Delta E_{j b}}\{\langle a b \mid i j\rangle-\langle a b \mid j i\rangle\} .
$$

From this, two important observations follow:

(i) The Hartree contribution $\langle a b \mid i j\rangle$ and the exchange term $-\langle a b \mid j i\rangle$ cancel each other for $j=i$ or for $a=b$ for any spin orientation. This self-correlation-error cancellation is obviously observed for all values of $\zeta$ since only same-spin electrons are affected by the self-correlation error. In other words, MP2 is self-correlation free and this property is shared in second order by the BSE. This property is also observed by the SOSEX approximation [25]. However, for SOSEX the corresponding second-order diagram is introduced in a somewhat ad hoc manner by antisymmetrizing the direct-RPA coupled-cluster amplitudes. For the BSE, the diagrams are naturally included at the level of the two-particle propagator and two-body density matrix. The BSE should therefore improve upon SOSEX; in particular if static correlation effects are important [31].

(ii) The expression for the correlation energy in second order also allows us to understand why the correlation energy converges so quickly with respect to the number of orbitals for the ferromagnetic case. For a ferromagnetic system, only one spin component is present. For large wave vectors, the 

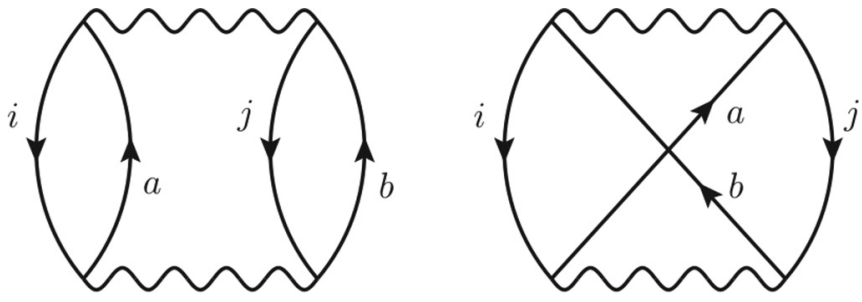

FIG. 4. Direct (left) and exchange (right) diagrams contributing in second order to the correlation energy.

dominant terms to the correlation energy stem from $i=j$, and the direct term is exactly canceled by the exchange term. This explains the fast convergence of the correlation energy with respect to the plane-wave cutoff for the ferromagnetic case. For a paramagnetic system, where both spin components are present, it is possible to carry out the summation over the spin degrees of freedom implicitly present in the spin-orbital representation above. This leads to a factor $2^{l}$, with $l$ being the number of fermionic loops in the diagrams in Fig. 4. Hence, if Eq. (26) is evaluated for spatial orbitals [73], a factor of four enters for the direct term, whereas a factor of two is present for the exchange term. Thus the two terms do not cancel each other in the long-wavelength limit, more precisely the direct term is reduced by a factor of two by the exchange term. This causes the slow convergence for the non-spin-polarized system.

Given the relevance of the HEG as a model for condensed matter, it is not surprising that other research groups have recently evaluated its properties as well. For instance, the use of the renormalized ALDA kernel with the inclusion of correlation effects seems to reproduce exactly the HEG correlation energy in the limit of very low densities $\left(r_{s}>10\right)$ [74]. However, larger deviations are observed in the "metallic" density region $\left(r_{s} \in[0.5,5.0]\right)$. Also, a recent study by the de Gironcoli group [75] has addressed the role of correlations in the HEG by employing exact exchange (EXX) timedependent density functional theory (TDDFT). A feature of this method is to include first-order changes of the one-particle propagator due to exact exchange, as well as particle-hole exchange interactions in the two-point response function (due to adiabatically switching on the exact exchange between electrons). This response function is then used to form the exchange kernel as proposed by Görling [76]. An issue that this approach faces is the occurrence of imaginary frequencies when the response function is diagonalized [see Eq. (10) in Ref. [75]]. Although it is possible to circumvent this problem by including the ladder diagrams (A2) and (B2) only to first order in the response function, this also spoils the results to some extent [75]. Other difficulties in the EXX-TDDFT method stem from the inversion of the noninteracting response function to evaluate the exchange kernel [see Eq. (7) in Ref. [23]] and on the critical dependence on the basis set size [77].

Also in the present BSE approach instabilities at lower electron densities can be present (as in our case for $r_{s} \geqslant 5$ ). These are witnessed as imaginary frequencies $\Omega$ appearing in Eq. (22). The instabilities can cause the matrices $\mathbf{A}+\mathbf{B}$ or $\mathbf{A}-\mathbf{B}$ to have negative eigenvalues. If both matrices have negative eigenvalues, it has been argued that the ground state is unstable with respect to particle-hole excitations [57]. In the present case, however, we find that only the A $+\mathbf{B}$ matrix has negative eigenvalues, for both the $G W_{0}$ and LDA reference states. This finding is consistent with a recent theoretical investigation [78] which has confirmed the presence of negative-frequency modes in the BSE polarization propagator.

In our case the instabilities originate from the particle-hole ladder diagrams, as also witnessed in the case of the alreadymentioned TDDFT calculations. To resolve this problem, we suggest disregarding all particle-hole ladder diagrams in the A matrix - specifically those shown in (A2) of Fig. 1-when solving the BSE equation. In the following, we refer to this approximation as RPA with screened exchange (RPAsX).

We believe this choice to be sensible for the following reasons: First, the A matrix does not contribute to the correlation energy in the perturbation expansion to second order $[58,79]$. Thus removing the diagrams (A2) will only change the correlation energy in third order; in second order, for instance, we still recover the exact MP2 energy (if the screened exchange kernel is replaced by the bare one). The approximation is also closely related to the AC-SOSEX method [25] but improves upon it. In the AC-SOSEX method, the conventional RPA polarization propagator is evaluated. As opposed to the direct RPA, the polarization propagator is then contracted against the $\mathbf{B}$ matrix [containing the sum of the direct Coulomb interaction and the bare exchange interaction, diagrams (B1) and (B2) in Eq. (23)] [18]. In RPAsX, the propagator now includes, as it should, the exchange term in the $\mathbf{B}$ matrix (B1 as well as B2). Furthermore, the exchange terms (B2) now include a screened exchange interaction instead of a bare interaction, which effectively mimics higher-order ladder diagrams. It is clear from Fig. 3 that this approximation is particularly successful for the HEG, because it completely prevents the occurrence of unstable solutions and yields excellent agreement with the QMC data for both spin-polarization values, as shown in Fig. 3 by a dash-dotted line. We observe a slight upward shift of the correlation energy compared with the full BSE approach, which leads to undercorrelation in comparison with CA by $0.03,0.02 \mathrm{eV}$ for $r_{s}=0.5$ and $0.8,1.0$ a.u., respectively. For $r_{s} \geqslant 2$ the RPAsX estimate of the correlation energy remains within $6 \%$ of the CA estimate for $\zeta=0$ (it overcorrelates to a greater extent in comparison with $\mathrm{OB}$ ). For the spin-polarized case, RPAsX lies within the range of values spanned by the different QMC simulations in the high-density region. As the density is lowered there is a progressive increase of the correlation energy in RPAsX, which still compares very favorably with CA values, reaching a maximum mismatch of $20 \mathrm{meV}$ for the lowest density considered. RPAsX correlation energy is mostly parallel to the BSE results, but moves closer to the exact results. We admit that the very good agreement with the QMC results must be fortuitous to some extend, since the RPAsX still neglects particle-particle and hole-hole ladder diagrams. But obviously the neglect of these diagrams cancels the alsoneglected particle-hole ladder diagram, at least for the HEG.

Finally, we consider a partially-spin-polarized system. The noninteracting response function is diagonal in the spin basis, and this property is conserved by the BSE kernel because opposite spin components interact only through the direct diagrams (A1) and (B1) in Fig. 1. As was mentioned, increasing 


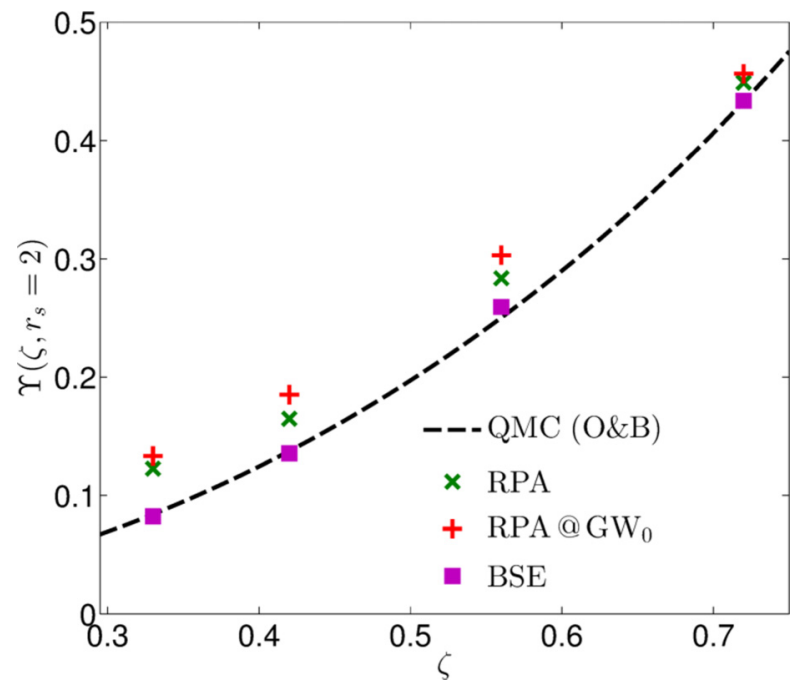

FIG. 5. The spin enhancement function $\Upsilon$ as a function of the fractional polarization at density $r_{s}=2$. Calculations were performed on a $6 \times 6 \times 6 \mathbf{k}$-point grid with complete basis set extrapolation.

the spin polarization decreases the number of electrons able to interact at short range. To assess the change introduced in the correlation energy $E_{c}$ by modifying the fraction of short-range vs long-range interactions, the spin-enhancement function $\Upsilon\left(\zeta ; r_{s}\right)$ is commonly introduced,

$$
\Upsilon\left(\zeta ; r_{s}\right)=\frac{E_{c}\left(\zeta, r_{s}\right)-E_{c}\left(0, r_{s}\right)}{E_{c}\left(1, r_{s}\right)-E_{c}\left(0, r_{s}\right)} .
$$

In Fig. 5 we report the spin enhancement function computed at $r_{s}=2$ versus the spin polarization $\zeta$ for various levels of theory. Results are compared against the Perdew-Wang interpolation formula [80] with parameters estimated by Ortiz and Ballone [69] (dashed line). RPA results are evaluated on the DFT reference state (represented in the figure by the " $X$ " symbols): there is a noticeable departure from the QMC estimate, with the RPA overestimating the short-range correlations, as expected. Changing reference state (RPA on $G W_{0}$ indicated by "+" symbols in the figure) actually increases the mismatch with the QMC estimate. The BSE results, shown by squares in the figure, reproduce exceedingly well the benchmark calculations for all values of $\zeta$ considered. Given the high computational cost required by these calculations we do not report RPAsX for fractional spin polarizations. However, since the BSE makes a more sizable error than RPAsX (see Fig. 3 ) and yet it reproduces $\Upsilon$ very accurately, it is reasonable to assume that RPAsX will also be very accurate for fractional values of $\zeta$.

\section{CONCLUSIONS}

In this study we have evaluated the correlation energy for the homogeneous electron gas by calculating the fluctuation contributions from the Bethe-Salpeter equation. The implementation of the BSE has taken advantage of the time-inversion symmetry, converting it into a Hermitian quadratic eigenvalue problem. Both the resonant and the antiresonant contributions to the particle-hole interaction have been included in the BSE (going beyond the so-called Tamm-Dancoff approximation).
The BSE kernel has been set up consistently with Hedin's $G W_{0}$ approximation. This level of theory has been employed also to describe the system's electronic structure, taken as a starting point for the ensuing BSE calculation. The occurrence of unstable solutions using the full Bethe-Salpeter kernel has prompted us to seek an approximation scheme that is still able to compare favorably with QMC references. The approximation proposed, called RPAsX, is by construction consistent with MP2 and reproduces the QMC correlation energy very well for values of $r_{s} \geqslant 1.0$ a.u. We certainly plan to test this approximation for a wider class of systems and materials. There are, however, several obstacles that need to be solved before this approach can be applied routinely. The most important one is that, for the HEG, changes related to changes of the one-particle Green's function and one-particle orbitals can be neglected [compare Eq. (4)]. This is not the case for real materials, where self-consistency of the orbitals [81] or changes of the one-particle Green's function when going to the interacting case are very relevant [44]. In order to apply the RPAsX to real systems it will be necessary to relax the approximations made here. With this provision, we are confident that this approach can be extended to realistic solid-state and molecular systems.

\section{ACKNOWLEDGMENTS}

Financial support from the Austrian Science Fund (FWF) for the SFB ViCoM Project No. F41 is kindly acknowledged.

\section{APPENDIX A: DERIVATION OF EQ. (5)}

In this Appendix we derive the expression for the correlation energy in terms of the polarization propagator. The correlation energy involves the two-body density matrix and, as shown in the main text for Eq. (4), is given by

$$
\begin{aligned}
\left(\frac{d E_{\lambda}}{d \lambda}\right)_{2 \mathrm{~B}} \equiv & \dot{E}_{\lambda}^{c}=\frac{1}{2} \int d t_{1} \int \prod_{i=1}^{4} d \mathbf{x}_{i} \delta\left(\mathbf{x}_{3}-\mathbf{x}_{1}\right) \\
& \times \delta\left(\mathbf{x}_{4}-\mathbf{x}_{2}\right) v\left(\mathbf{x}_{1}, \mathbf{x}_{2}\right) \\
& \times\left\langle\psi^{\dagger}\left(\mathbf{x}_{4}, t_{1}^{+}\right) \psi^{\dagger}\left(\mathbf{x}_{3}, t_{1}\right) \psi\left(\mathbf{x}_{1}, t_{1}^{+}\right) \psi\left(\mathbf{x}_{2}, t_{1}\right)\right\rangle_{\lambda} .
\end{aligned}
$$

The four field operators in this expression suggest that one can make a connection to Green's function theory, specifically to the two-particle Green's function. We also note that the expression above only involves equal time limits (all involved time points are equal). To make a connection to Green's function theory, we first need to introduce a time dependence in the field operators by defining the time evolution operator in the interaction picture (as is commonly done in many-body perturbation theory): $\hat{\psi}(1)=e^{i \mathcal{H}_{0} t_{1}} \hat{\psi}\left(\mathbf{x}_{1}\right) e^{-i \mathcal{H}_{0} t_{1}}$ and $\hat{\psi}^{\dagger}(2)=$ $e^{i \mathcal{H}_{0} t_{2}} \hat{\psi}^{\dagger}\left(\mathbf{x}_{2}\right) e^{-i \mathcal{H}_{0} t_{2}}$. The $n$-body Green's operator is as usually defined as

$$
\begin{aligned}
& \mathcal{G}_{n}\left(1,2, \ldots, n ; 1^{\prime}, 2^{\prime}, \ldots, n^{\prime}\right) \\
& \quad=\frac{1}{i^{n}} \mathcal{T}\left\{\hat{\psi}(1) \hat{\psi}(2) \cdots \hat{\psi}(n) \hat{\psi}^{\dagger}\left(n^{\prime}\right) \cdots \hat{\psi}^{\dagger}\left(2^{\prime}\right) \hat{\psi}^{\dagger}\left(1^{\prime}\right)\right\},
\end{aligned}
$$

where $\mathcal{T}$ is the time-ordering operator. It is worth pointing out that the operators $\mathcal{G}_{n}$ do not depend on the value of the 
coupling constant $\lambda$. For $n=1$ the corresponding (greater) Green's function is obtained by evaluating the expectation value of the Green's operator for the ground-state wave function at coupling $\lambda, \Psi_{\lambda}$. Since we are only interested in equal time limits here, we can restrict the second time to fulfill $t^{\prime}=t+0^{+}=t^{+}$:

$$
\begin{aligned}
\left\langle\mathcal{G}_{1}\left(\mathbf{x}_{1}, t^{+} ; \mathbf{x}_{2}, t\right)\right\rangle_{\lambda} & \equiv G_{1, \lambda}\left(\mathbf{x}_{1}, t^{+} ; \mathbf{x}_{2}, t\right) \\
& =i\left\langle\psi^{\dagger}\left(\mathbf{x}_{2}, t\right) \psi\left(\mathbf{x}_{1}, t^{+}\right)\right\rangle_{\lambda} .
\end{aligned}
$$

At equal time limits, the Green's function can be also related to the density matrix $\langle\hat{n}\rangle_{\lambda} \equiv n_{\lambda}(\mathbf{x}, \mathbf{y})=-i G_{1, \lambda}(\mathbf{x}, t ; \mathbf{y}, t)$.

For the two-particle Green's function, it is standard textbook material [47] (commutator relations or Wick's theorem) to show that it satisfies

$$
\begin{aligned}
G_{2, \lambda} & \left(\mathbf{x}_{1}, t_{1}^{+}, \mathbf{x}_{2}, t_{2} ; \mathbf{x}_{3}, t_{1}, \mathbf{x}_{4}, t_{2}^{+}\right) \\
= & -\left\langle\psi^{\dagger}\left(\mathbf{x}_{3}, t_{1}\right) \psi\left(\mathbf{x}_{1}, t_{1}^{+}\right)\right\rangle_{\lambda}\left\langle\psi^{\dagger}\left(\mathbf{x}_{4}, t_{2}^{+}\right) \psi\left(\mathbf{x}_{2}, t_{2}\right)\right\rangle_{\lambda} \\
& +\left\langle\psi^{\dagger}\left(\mathbf{x}_{4}, t_{2}^{+}\right) \psi\left(\mathbf{x}_{1}, t_{1}^{+}\right)\right\rangle_{\lambda}\left\langle\psi^{\dagger}\left(\mathbf{x}_{3}, t_{1}\right) \psi\left(\mathbf{x}_{2}, t_{2}\right)\right\rangle_{\lambda} \\
& +\left\langle\psi^{\dagger}\left(\mathbf{x}_{4}, t_{2}^{+}\right) \psi^{\dagger}\left(\mathbf{x}_{3}, t_{1}\right) \psi\left(\mathbf{x}_{1}, t_{1}^{+}\right) \psi\left(\mathbf{x}_{2}, t_{2}\right)\right\rangle_{\lambda},
\end{aligned}
$$

where the last term on the right-hand side is the correlation part of the two-body Green's function that is also present in the AC fluctuation-dissipation theorem (ACFDT) correlation energy (A1). The labeling convention for four-point quantities is given in Fig. 6 and is consistent with Ref. [82] up to a time inversion. Since the correlation energy involves only equal time limits, we again set $t_{1}=t_{2}=t$. This allows us to rewrite Eq. (A4) in terms of the ordinary density operators $\hat{n}$ and their expectation values:

$$
\begin{aligned}
G_{2, \lambda} & \left(\mathbf{x}_{1}, t^{+}, \mathbf{x}_{2}, t ; \mathbf{x}_{3}, t, \mathbf{x}_{4}, t^{+}\right) \\
= & -\left\langle\psi^{\dagger}\left(\mathbf{x}_{3}, t\right) \psi\left(\mathbf{x}_{1}, t^{+}\right)\right\rangle_{\lambda}\left\langle\psi^{\dagger}\left(\mathbf{x}_{4}, t^{+}\right) \psi\left(\mathbf{x}_{2}, t\right)\right\rangle_{\lambda} \\
& +n_{\lambda}\left(\mathbf{x}_{4}, \mathbf{x}_{1}\right) n_{\lambda}\left(\mathbf{x}_{3}, \mathbf{x}_{2}\right)-\left\langle\hat{n}\left(\mathbf{x}_{4}, \mathbf{x}_{1}\right) \hat{n}\left(\mathbf{x}_{3}, \mathbf{x}_{2}\right)\right\rangle_{\lambda} \\
= & -\left\langle\psi^{\dagger}\left(\mathbf{x}_{3}, t\right) \psi\left(\mathbf{x}_{1}, t^{+}\right)\right\rangle_{\lambda}\left\langle\psi^{\dagger}\left(\mathbf{x}_{4}, t^{+}\right) \psi\left(\mathbf{x}_{2}, t\right)\right\rangle_{\lambda} \\
& -\left\langle\delta \hat{n}\left(\mathbf{x}_{4}, \mathbf{x}_{1}\right) \delta \hat{n}\left(\mathbf{x}_{3}, \mathbf{x}_{2}\right)\right\rangle_{\lambda},
\end{aligned}
$$

where, in going to the last line, we introduced the density fluctuation operator $\langle\delta \hat{n}\rangle_{\lambda} \equiv\langle\hat{n}\rangle_{\lambda}-n_{\lambda}$. The last term in Eq. (A5) can be identified with the polarization propagator (also called density-fluctuation density-fluctuation response function), i.e., $P_{\lambda} \equiv\langle\delta \hat{n} \delta \hat{n}\rangle_{\lambda}$. One can then rearrange Eq. (A5) into the usual relation connecting the two-particle propagator and the polarization propagator [7]:

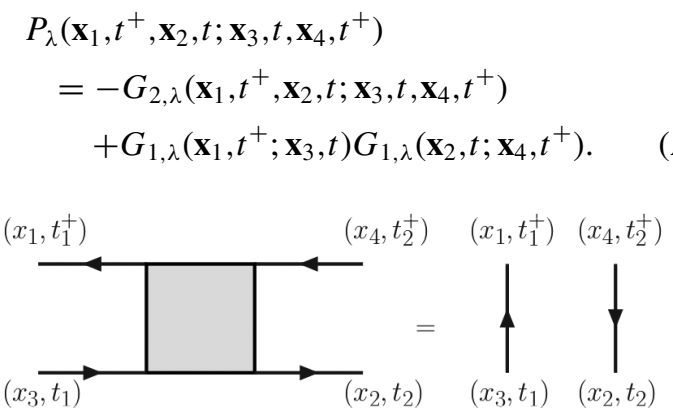

By using Eqs. (A4) and (A6), one can rewrite the correlation part of $G_{2, \lambda}$ in the ACFDT equation by using the polarization propagator and obtain the following compact equation for the correlation energy:

$$
\begin{aligned}
\dot{E}_{\lambda}^{\mathrm{c}}= & \frac{1}{2} \int \prod_{i=1}^{4} d \mathbf{x}_{i} V\left[G_{1, \lambda}\left(\mathbf{x}_{1}, t^{+} ; \mathbf{x}_{4}, t^{+}\right) G_{1, \lambda}\left(\mathbf{x}_{2}, t ; \mathbf{x}_{3}, t\right)\right. \\
& \left.-P_{\lambda}\left(\mathbf{x}_{1}, t^{+}, \mathbf{x}_{2}, t ; \mathbf{x}_{3}, t, \mathbf{x}_{4}, t^{+}\right)\right] .
\end{aligned}
$$

From Eq. (A7) it is easy to separate out the exchange contribution:

$$
\begin{aligned}
\dot{E}_{\lambda}^{\mathrm{x}}= & -\frac{1}{2} \int \prod_{i=1}^{4} d \mathbf{x}_{i} \delta\left(\mathbf{x}_{3}-\mathbf{x}_{1}\right) \delta\left(\mathbf{x}_{4}-\mathbf{x}_{2}\right) \\
& \times v\left(\mathbf{x}_{1}, \mathbf{x}_{2}\right) n_{\lambda}\left(\mathbf{x}_{2}, \mathbf{x}_{3}\right) n_{\lambda}\left(\mathbf{x}_{1}, \mathbf{x}_{4}\right) \\
= & -\frac{1}{2} \int d \mathbf{x}_{1} d \mathbf{x}_{2} v\left(\mathbf{x}_{1}, \mathbf{x}_{2}\right) n_{\lambda}\left(\mathbf{x}_{2}, \mathbf{x}_{1}\right) n_{\lambda}\left(\mathbf{x}_{1}, \mathbf{x}_{2}\right) .
\end{aligned}
$$

The remaining contribution in Eq. (A7) can be replaced into Eq. (3) to form the correlation energy assuming that the onebody contributions (contained in the exchange part) remain constant along the AC path. Equation (5) is then obtained by Fourier transforming with respect to the infinitesimal time difference.

\section{APPENDIX B: SPECTRAL REPRESENTATION FOR THE POLARIZATION PROPAGATOR}

The starting point to derive the spectral representation for the interacting polarization propagator $P_{\lambda}(\omega)$ is to invert Eq. (8) in the particle-hole basis:

$$
\mathbf{P}_{\lambda}(\omega)=\left[\mathbf{P}_{0}^{-1}(\omega)-\mathbf{I}_{\lambda}\right]^{-1} .
$$

We note that, for real matrices, an analogous but formally somewhat different presentation can be found in Furche [83]. Poles in the polarization propagator will be located at those frequencies that fulfill the condition $\operatorname{det}\left\{\mathbf{P}_{0}^{-1}(\omega)-\mathbf{I}_{\lambda}\right\}=0$. Given the spectral representation for $\mathbf{P}_{\mathbf{0}}(\omega)$ in Eq. (9) it is straightforward to construct its inverse, both being diagonal matrices:

$$
\begin{aligned}
\mathbf{P}_{0}^{-1}(\omega) & =\left(\begin{array}{cc}
-\Delta E_{i a} & \mathbf{0} \\
\mathbf{0} & \Delta E_{i a}
\end{array}\right)+\omega\left(\begin{array}{cc}
\mathbf{1} & \mathbf{0} \\
\mathbf{0} & -\mathbf{1}
\end{array}\right) \\
& =\mathbf{D}+\omega \boldsymbol{\Delta} .
\end{aligned}
$$

In going to the last line we introduced the supermatrices $\mathbf{D}$ and $\boldsymbol{\Delta}$ for convenience. The polarization propagator can then be compactly expressed as

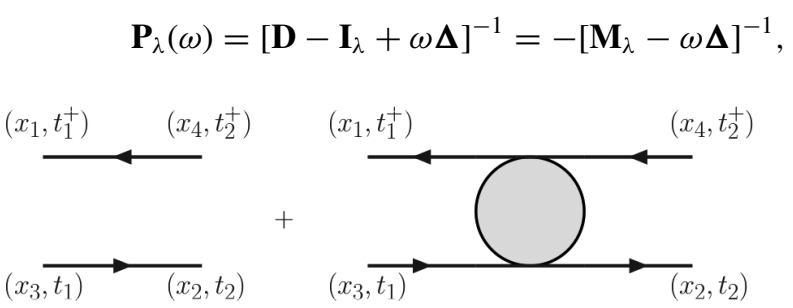

FIG. 6. Graphical representation for the two-body Green's function. We represent the time ordering $t_{1}=t_{2}$, which corresponds to having two electron-hole pairs interacting instantaneously. 
where the square supermatrix $\mathbf{M}_{\lambda}$ corresponds to the one introduced in Eq. (10). Since the determinant is invariant under similarity transformations, it is convenient to introduce the matrix

$$
\mathbf{Z}_{\lambda}=\left(\begin{array}{ll}
\mathbf{X}_{\lambda} & \mathbf{Y}_{\lambda}^{*} \\
\mathbf{Y}_{\lambda} & \mathbf{X}_{\lambda}^{*}
\end{array}\right)
$$

that fulfils the generalized eigenvalue equation

$$
\mathbf{M}_{\lambda} \mathbf{Z}_{\lambda}=\Delta \mathbf{Z}_{\lambda} \Omega_{\lambda},
$$

which is nothing but a restatement of Eq. (10) taking into account the complex conjugate solution. The matrix $\mathbf{Z}_{\lambda}$ satisfies the "symplectic" normalization condition $\mathbf{Z}_{\lambda} \boldsymbol{\Delta} \mathbf{Z}_{\lambda}^{\dagger}=$ $\boldsymbol{\Delta}$; this constraint, together with $\boldsymbol{\Delta}$ being idempotent, are sufficient to show that $\mathbf{Z}_{\lambda}$ is unitary, and that the condition

$$
\Delta \mathbf{Z}_{\lambda}^{\dagger} \boldsymbol{\Delta}=\mathbf{Z}_{\lambda}^{-1}
$$

holds.
The relation in Eq. (B5) can be exploited to construct the spectral representation of the polarization propagator starting from Eq. (B4):

$$
\begin{aligned}
& \left\{\mathbf{M}_{\lambda}-\omega \boldsymbol{\Delta}\right\} \mathbf{Z}_{\lambda}=\boldsymbol{\Delta} \mathbf{Z}_{\lambda}\left(\Omega_{\lambda}-\omega\right), \\
\Rightarrow & \mathbf{M}_{\lambda}-\omega \boldsymbol{\Delta}=\boldsymbol{\Delta} \mathbf{Z}_{\lambda}\left(\Omega_{\lambda}-\omega\right) \boldsymbol{\Delta} \mathbf{Z}_{\lambda}^{\dagger} \boldsymbol{\Delta} .
\end{aligned}
$$

Inverting the right-hand side of the previous equation, one finally obtains

$$
\mathbf{P}_{\lambda}(\omega)=\mathbf{Z}_{\lambda}\left(\omega-\Omega_{\lambda}\right)^{-1} \boldsymbol{\Delta} \mathbf{Z}_{\lambda}^{\dagger}
$$

The presence of a simple pole analytic structure for both polarization propagators entering Eq. (5) is of fundamental importance because it allows the straightforward frequency integration necessary to evaluate the correlation part of the two-body density matrix $\mathcal{P}_{\lambda}$.
[1] G. Giuliani and G. Vignale, Quantum Theory of the Electron Liquid (Cambridge University Press, Cambridge, New York, 2005).

[2] T. Endo, M. Horiuchi, Y. Takada, and H. Yasuhara, Phys. Rev. B 59, 7367 (1999).

[3] J. Hubbard, Proc. R. Soc. London, Ser. A 244, 199 (1958).

[4] P. Gori-Giorgi, F. Sacchetti, and G. B. Bachelet, Phys. Rev. B 61, 7353 (2000).

[5] K. Sturm and A. Gusarov, Phys. Rev. B 62, 16474 (2000).

[6] F. Caruso, D. R. Rohr, M. Hellgren, X. Ren, P. Rinke, A. Rubio, and M. Scheffler, Phys. Rev. Lett. 110, 146403 (2013).

[7] G. Stefanucci and R. van Leeuwen, Nonequilibrium ManyBody Theory of Quantum Systems: A Modern Introduction (Cambridge University Press, Cambridge, New York, 2013).

[8] F. Furche, Phys. Rev. B 64, 195120 (2001).

[9] T. Miyake, F. Aryasetiawan, T. Kotani, M. van Schilfgaarde, M. Usuda, and K. Terakura, Phys. Rev. B 66, 245103 (2002).

[10] Y. M. Niquet, M. Fuchs, and X. Gonze, Phys. Rev. A 68, 032507 (2003).

[11] H. Jiang and E. Engel, J. Chem. Phys. 127, 184108 (2007).

[12] F. Furche, J. Chem. Phys. 129, 114105 (2008).

[13] G. E. Scuseria, T. M. Henderson, and D. C. Sorensen, J. Chem. Phys. 129, 231101 (2008).

[14] J. Harl and G. Kresse, Phys. Rev. B 77, 045136 (2008).

[15] J. Harl, L. Schimka, and G. Kresse, Phys. Rev. B 81, 115126 (2010).

[16] J. Toulouse, I. C. Gerber, G. Jansen, A. Savin, and J. G. Ángyán, Phys. Rev. Lett. 102, 096404 (2009).

[17] J. Toulouse, W. Zhu, J. G. Ángyán, and A. Savin, Phys. Rev. A 82, 032502 (2010).

[18] G. Jansen, R.-F. Liu, and J. G. Ángyán, J. Chem. Phys. 133, 154106 (2010).

[19] T. Olsen and K. S. Thygesen, Phys. Rev. B 87, 075111 (2013).

[20] A. Heßelmann and A. Görling, Mol. Phys. 108, 359 (2010).

[21] M. Macher, J. Klimeš, C. Franchini, and G. Kresse, J. Chem. Phys. 140, 084502 (2014).
[22] A. M. Teale, S. Coriani, and T. Helgaker, J. Chem. Phys. 132, 164115 (2010).

[23] P. Bleiziffer, A. Heßelmann, and A. Görling, J. Chem. Phys. 136, 134102 (2012).

[24] M. Kaltak, J. Klimeš, and G. Kresse, J. Chem. Theory Comput. 10, 2498 (2014).

[25] A. Grüneis, M. Marsman, J. Harl, L. Schimka, and G. Kresse, J. Chem. Phys. 131, 154115 (2009).

[26] L. Schimka, J. Harl, A. Stroppa, A. Grüneis, M. Marsman, F. Mittendorfer, and G. Kresse, Nat. Mater. 9, 741 (2010).

[27] P. García-González, J. J. Fernández, A. Marini, and A. Rubio, J. Phys. Chem. A 111, 12458 (2007).

[28] J. Harl and G. Kresse, Phys. Rev. Lett. 103, 056401 (2009).

[29] X. Ren, A. Tkatchenko, P. Rinke, and M. Scheffler, Phys. Rev. Lett. 106, 153003 (2011).

[30] P. Mori-Sánchez, A. J. Cohen, and W. Yang, Phys. Rev. A 85, 042507 (2012).

[31] T. M. Henderson and G. E. Scuseria, Mol. Phys. 108, 2511 (2010).

[32] T. Olsen and K. S. Thygesen, J. Chem. Phys. 140, 164116 (2014).

[33] S. Albrecht, L. Reining, R. Del Sole, and G. Onida, Phys. Rev. Lett. 80, 4510 (1998).

[34] M. Rohlfing and S. G. Louie, Phys. Rev. B 62, 4927 (2000).

[35] J. Yan, K. W. Jacobsen, and K. S. Thygesen, Phys. Rev. B 86, 045208 (2012).

[36] M. Gatti and F. Sottile, Phys. Rev. B 88, 155113 (2013).

[37] M. L. del Puerto, M. L. Tiago, and J. Chelikowsky, Phys. Rev. Lett. 97, 096401 (2006).

[38] I. Duchemin and X. Blase, Phys. Rev. B 87, 245412 (2013).

[39] J. Linderberg, Int. J. Quantum Chem. 1, 719 (1967).

[40] O. Gunnarsson and B. Lundqvist, Phys. Rev. B 13, 4274 (1976).

[41] D. C. Langreth and J. P. Perdew, Phys. Rev. B 15, 2884 (1977).

[42] J. Harris and R. O. Jones, J. Phys. F: Met. Phys. 4, 1170 (1974).

[43] K. S. Singwi, M. Tosi, R. Land, and A. Sjölander, Phys. Rev. 176, 589 (1968).

[44] J. Klimeš, M. Kaltak, E. Maggio, and G. Kresse, J. Chem. Phys. 143, 102816 (2015).

[45] J. Harris, Phys. Rev. A 29, 1648 (1984). 
[46] H. van Aggelen, Y. Yang, and W. Yang, J. Chem. Phys. 140, 18A511 (2014).

[47] E. K. U. Gross, E. Runge, and O. Heinonen, Many-Particle Theory (IOP Publishing, Bristol, Philadelphia, New York, 1991).

[48] K. Toyoda, T. Iwai, and O. Tanimoto, Prog. Theor. Phys. 54, 333 (1975).

[49] A. L. Fetter and J. D. Walecka, Quantum Theory of ManyParticle Systems (McGraw-Hill, Inc., New York, 1971), p. 601.

[50] G. Strinati, La Riv. del Nuovo Cimento 11, 1 (1988).

[51] W. Hanke and L. J. Sham, Phys. Rev. Lett. 43, 387 (1979).

[52] W. Hanke and L. J. Sham, Phys. Rev. B 21, 4656 (1980).

[53] F. Fuchs, C. Rödl, A. Schleife, and F. Bechstedt, Phys. Rev. B 78, 085103 (2008).

[54] T. Sander, E. Maggio, and G. Kresse, Phys. Rev. B 92, 045209 (2015).

[55] C. Rödl, F. Fuchs, J. Furthmüller, and F. Bechstedt, Phys. Rev. B 77, 184408 (2008).

[56] P. Ring and P. Schuck, The Nuclear Many-Body Problem, 3rd ed. (Springer-Verlag, Berlin, Heidelberg, 1980).

[57] P. Jørgensen and J. Simons, Second-Quantization-Based Methods in Quantum Chemistry (Academic Press, New York, 1981).

[58] J. G. Ángyán, R.-F. Liu, J. Toulouse, and G. Jansen, J. Chem. Theory Comput. 7, 3116 (2011).

[59] C. Lin, F. H. Zong, and D. M. Ceperley, Phys. Rev. E 64, 016702 (2001).

[60] G. Kresse and J. Furthmüller, Comput. Mater. Sci. 6, 15 (1996).

[61] G. Kresse and J. Furthmüller, Phys. Rev. B 54, 11169 (1996).

[62] M. Shishkin and G. Kresse, Phys. Rev. B 75, 235102 (2007).

[63] M. Shishkin and G. Kresse, Phys. Rev. B 74, 035101 (2006).
[64] P. Gori-Giorgi and J. P. Perdew, Phys. Rev. B 66, 165118 (2002).

[65] J. Klimeš, M. Kaltak, and G. Kresse, Phys. Rev. B 90, 075125 (2014).

[66] J. P. Perdew and A. Zunger, Phys. Rev. B 23, 5048 (1981).

[67] M. Marsman, A. Grüneis, J. Paier, and G. Kresse, J. Chem. Phys. 130, 184103 (2009).

[68] D. M. Ceperley and B. J. Alder, Phys. Rev. Lett. 45, 566 (1980).

[69] G. Ortiz and P. Ballone, Phys. Rev. B 50, 1391 (1994).

[70] L. M. Fraser, W. M. C. Foulkes, G. Rajagopal, R. J. Needs, S. D. Kenny, and A. J. Williamson, Phys. Rev. B 53, 1814 (1996).

[71] H. Yasuhara, Solid State Commun. 11, 1481 (1972).

[72] J. Paier, X. Ren, P. Rinke, G. E. Scuseria, A. Grüneis, G. Kresse, and M. Scheffler, New J. Phys. 14, 043002 (2012).

[73] J. Paldus and J. Čížek, Adv. Quantum Chem. 9, 105 (1975).

[74] C. E. Patrick and K. S. Thygesen, J. Chem. Phys. 143, 102802 (2015).

[75] N. Colonna, M. Hellgren, and S. de Gironcoli, Phys. Rev. B 90, 125150 (2014).

[76] A. Görling, Int. J. Quantum Chem. 69, 265 (1998).

[77] S. Hirata, S. Ivanov, I. Grabowski, R. J. Bartlett, K. Burke, and J. D. Talman, J. Chem. Phys. 115, 1635 (2001).

[78] A.-M. Uimonen, G. Stefanucci, Y. Pavlyukh, and R. van Leeuwen, Phys. Rev. B 91, 115104 (2015).

[79] A. Heßelmann and A. Görling, Mol. Phys. 109, 2473 (2011).

[80] J. P. Perdew and Y. Wang, Phys. Rev. B 45, 13244 (1992).

[81] M. van Schilfgaarde, T. Kotani, and S. Faleev, Phys. Rev. Lett. 96, 226402 (2006).

[82] R. Starke and G. Kresse, Phys. Rev. B 85, 075119 (2012).

[83] F. Furche, J. Chem. Phys. 114, 5982 (2001). 